J. L. Dupont

Nagoya Math. J.

Vol. 114 (1989), 1-20

\title{
ON POLYLOGARITHMS*)
}

\author{
JOHAN L. DUPONT
}

\section{§ 0. Introduction}

Some functions related to the complex dilogarithmic function

$$
\operatorname{Li}_{2}(z)=\sum_{m=1}^{\infty} \frac{z^{m}}{m^{2}}, \quad|z|<1
$$

(in the notation of Lewin [9]) are known to occur in connection with algebraic $K$-theory and characteristic classes (see e.g. Bloch [1], GelfandMacPherson [7], Dupont [5], and the references given there). Recently MacPherson and Hain (see [10]) has announced results of a similar kind for some higher polylogarithmic functions. Also Ramakrishnan [11] and [12] has recently studied the classical polylogarithms, which for $|z|<1$ are given by

$$
\operatorname{Li}_{n}(z)=\sum_{m=1}^{\infty} \frac{z^{m}}{m^{n}}
$$

In this note we shall pursue an idea in Bloch [1] and [2] where the dilogarithm takes values in the tensor-product (over $\mathbb{Z}$ ) $\mathbb{C} \otimes \mathbb{C}^{*}$, with $\mathbb{C}$ and $\mathbb{C}^{*}=\mathbb{C}-\{0\}$ being respectively the additive and multiplicative group of complex numbers. Thus let

$$
L_{n}^{*}: \mathbb{C} \backslash\{0,1\} \longrightarrow \mathbb{C}^{*} \otimes \cdots \otimes \mathbb{C}^{*} \quad(n \text { copies })
$$

be given by

$$
L_{n}^{*}(z)=(1-z) \otimes z \otimes \cdots \otimes z \quad\left(n-1 z^{\prime} \mathrm{s}\right)
$$

and consider the exponential map $e: \mathbb{C} \otimes \cdots \otimes \mathbb{C} \rightarrow \mathbb{C}^{*} \otimes \cdots \otimes \mathbb{C}^{*}$ (0.4) $\quad e\left(a_{1} \otimes \cdots \otimes a_{n}\right)=\exp \left(2 \pi i a_{1}\right) \otimes \cdots \otimes \exp \left(2 \pi i a_{n}\right), \quad a_{1}, \cdots, a_{n} \in \mathbb{C}$.

*) This work is partially supported by grants from Statens Naturvidenskabelige Forskningsråd, Denmark, and the National Science Foundation, U.S.A. 
Then we shall define a natural lift $L_{n}$ in the diagram

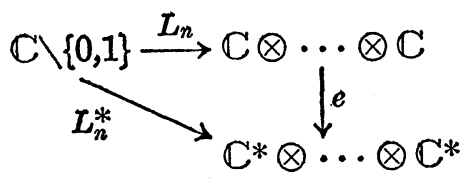

where $L_{n}$ is an expression involving the polylogarithms $\mathrm{Li}_{1}, \cdots, \mathrm{Li}_{n}$. The explicit formula is given in Corollary 4.7. However, a lift in (0.5) is not unique. Thus e.g. for $n=2$ one can add terms of the form

$$
\frac{\log z}{2 \pi i} \otimes 1-1 \otimes \frac{\log z}{2 \pi i}
$$

without changing the image in $\mathbb{C}^{*} \otimes \mathbb{C}^{*}$. One might therefore ask what motivates our particular choice. First of all the formula for $L_{n}$ is a natural generalization of the dilogarithm as it occurs in [1] or [5] and, in spite of the length of the formula, it seems difficult to produce anything shorter which is well-defined. More importantly there is a certain recursiveness in the formula for $L_{n}$ : If one considers the reduction of $L_{n}$ in the group

$$
\mathbb{C} \otimes \cdots \otimes \mathbb{C} \mathbb{Q} \otimes \cdots \otimes \mathbb{C} \mathbb{Q} \otimes \cdots \otimes \mathbb{C}
$$

(where $\mathbb{C}$ is reduced to $\mathbb{C} / \mathbb{Q}$ in two places) then the image is a tensor product of 2 similar formulas of lower order (formula (5.14) below). This can be exploited when one tries to prove relations for these polylogarithms. It clearly follows from the diagram (0.5) that a relation involving $L_{n}$ 's must necessarily be satisfied by the algebraic symbols $L_{n}^{*}$. We show that one can also work backwards - at least one can reduce the proof of such a relation to some involving only polylogarithms of lower order. As an example we mention 2 relations among trilogarithms which has occurred in our work with C.-H. Sah [6] on the homology of the discrete group $\operatorname{Gl}(n, \mathbb{C})$. (See also [14, Section 4].) There the algebraically defined maps $L_{n}^{*}$ naturally occurs in expressions of certain differentials in a spectral sequence. It is expected that the analogous expressions involving the lifts $L_{n}$ should also give homological invariants for $\operatorname{Gl}(n, \mathbb{C})$ similar to the situation for the dilogarithm in the case $n=2$.

Finally let us observe that we obtain well-defined real-valued polylogarithmic functions as in Ramakrishnan [12] if we compose $L_{n}$ with one of the $n$ maps $\mathbb{C} \otimes \cdots \otimes \mathbb{C} \rightarrow \mathbb{R}$ defined by 


$$
a_{1} \otimes \cdots \otimes a_{n} \longrightarrow\left(\operatorname{Re} a_{1}\right) \cdots\left(\operatorname{Re} a_{i-1}\right)\left(\operatorname{Im} a_{i}\right)\left(\operatorname{Re} a_{i+1}\right) \cdots\left(\operatorname{Re} a_{n}\right)
$$

$(i=1, \cdots, n)$ where $\operatorname{Re}$ and Im denotes the real and imaginary parts.

\section{$\S 1$. Iterated integrals}

Polylogarithms are defined by iterated integrals in the sense of K.-T. Chen (see [3] and the references given there; see also Hain [8]). Thus let $M$ be a Riemann surface (e.g. $M=\mathbb{C}-\left\{z_{1}, \cdots, z_{m}\right\}$ ) and $\omega_{1}, \cdots, \omega_{n}$ some holomorphic 1-forms (not necessarily different) on $M$. Let $\pi: \tilde{M} \rightarrow M$ be the universal covering and $z_{0} \in \tilde{M}$ a base point. The iterated integral is defined inductively as a solution on $\tilde{M}$ to the differential equation

$$
d \int \omega_{1} \cdots \omega_{n}=\left(\int \omega_{1} \cdots \omega_{n-1}\right) \omega_{n} .
$$

More precisely given initial values $c_{1}, \cdots, c_{n} \in \mathbb{C}$ the vector $\underline{a}=\left(1, a_{1}, \cdots, a_{n}\right)$ with

$$
a_{j}=\int \omega_{1} \cdots \omega_{j}
$$

is a solution to the system of differential equations

$$
\begin{aligned}
& d a_{j}=a_{j-1} \omega_{j}, \quad j=1, \cdots, n, \quad\left(a_{0}=1\right) \\
& a_{j}\left(z_{0}\right)=c_{j} .
\end{aligned}
$$

Explicitly, if $\gamma$ is a path in $\tilde{M}$ from $z_{0}$ to $z \in \tilde{M}$ then

$$
a_{j}(z)=\int^{z} \omega_{1} \cdots \omega_{j}=\sum_{i=0}^{j-1} c_{i} \int_{\gamma} \omega_{i+1} \cdots \omega_{j}+c_{j} \quad\left(c_{0}=1\right)
$$

where $\int_{r} \omega_{i+1} \cdots \omega_{j}, i<j$, are iterated path-integrals as in Chen [3, Ch. 1]. Thus we are here using the notation $\int \omega_{1} \cdots \omega_{n}$ (or $\int^{z} \omega_{1} \cdots \omega_{n}$ when we indicate the variable $z$ ) slightly more generally than used by Chen. Therefore in the following when specifying $\int \omega_{1} \cdots \omega_{n}$ it is necessary also to consider all the integrals $\int m_{i+1} \cdots \omega_{j}, 0 \leqq i<j \leqq n$ at the same time:

Let $N(n, \mathbb{C}) \subseteq \mathrm{Gl}(n+1, \mathbb{C})$ be the subgroup of unipotent matrices $A=\left(a_{i j}\right)$ with $a_{i i}=1, a_{i j}=0$ if $j<i$. Then given $C=\left(c_{i j}\right) \in N(n, \mathbb{C})$ we have a unique holomorphic solution $A=A(z), z \in \tilde{M}$, to the matrix equation 


$$
\begin{aligned}
& d A=A \Omega \\
& A\left(z_{0}\right)=C
\end{aligned}
$$

where $\Omega$ is the $(n+1) \times(n+1)$ matrix

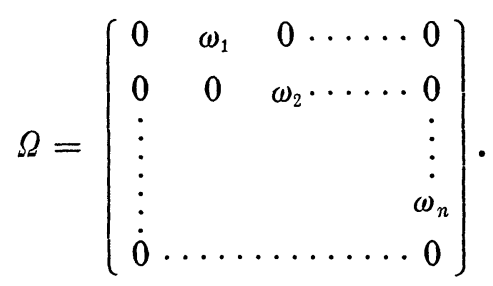

Then $a_{i j}=\int \omega_{i+1} \cdots \omega_{j}, i<j$ with initial condition $a_{i j}\left(z_{0}\right)=c_{i j} . \quad$ In particular for $r$ a path in $\tilde{M}$ starting at $z_{0}$ we denote the solution to (1.5) along $\gamma$ with initial value $A\left(z_{0}\right)=I$ by $A_{r}$. If the endpoint of $\gamma$ is $z$ then clearly the solution to (1.5) is given by

$$
A(z)=C A_{r} .
$$

It follows that for two composable paths $\alpha$ and $\beta$ we have for the composite path $\alpha * \beta$ (first $\alpha$ then $\beta$ ):

$$
A_{a * \beta}=A_{\alpha} A_{\beta} .
$$

This is equivalent to the equation in Chen $[3,1.6 .1$.

$$
\int_{\alpha * \beta} \omega_{i+1} \cdots \omega_{j}=\sum_{s=i}^{j}\left(\int_{\alpha} \omega_{i+1} \cdots \omega_{s}\right)\left(\int_{\beta} \omega_{s+1} \cdots \omega_{j}\right) \quad 0 \leqq i<j \leqq n .
$$

In particular $\gamma \mapsto A_{r}$ defines a homomorphism of the fundamental group $\pi_{1}\left(M, \pi z_{0}\right)$ to $N(n, \mathbb{C})$. Let $\Gamma_{z_{0}} \subseteq N(n, \mathbb{C})$ be the image. Then for $\alpha$ a closed curve in $M$ at $\pi\left(z_{0}\right)$ the lift of $\alpha$ starting at $z_{0}$ defines the monodromy transformation $A_{\alpha} \in \Gamma_{z_{0}}$. It follows from (1.7) that if $g$ denotes the covering transformation of $\tilde{M}$ corresponding to $\alpha$ then the solution to (1.5) satisfies

$$
A\left(g_{\alpha}(z)\right)=C A_{\alpha} C^{-1} A(z) .
$$

Hence $A$ gives a well-defined holomorphic mapping

$$
A: M \longrightarrow C \Gamma_{z_{0}} C^{-1} \backslash N(n, \mathbb{C}) \text {. }
$$

It is useful to see how the monodromy transformation change with change of basepoint. Thus for $g$ a covering transformation of $\tilde{M}$ and $z \in \tilde{M}$ let $\Lambda_{g}(z) \in N(n, \mathbb{C})$ be the monodromy transformation for a curve 
starting at $z$ and representing $g$ in $\pi_{1}(M, \pi z)$. That is, if $z=z_{0}$ and $g$ is represented by $\alpha$ then $\Lambda_{g}\left(z_{0}\right)=A_{\alpha}$ in the above notation. We now have

Proposition 1.12. i) $\Lambda_{g}$ is a holomorphic solution to the equation

$$
d \Lambda=[\Lambda, \Omega]
$$

ii) If $A$ is a solution to (1.5) and $A$ is a solution to (1.13) then $A \Lambda A^{-1}$ is constant, i.e.,

$$
A(z) \Lambda(z) A^{-1}(z) \equiv C A_{\alpha} C^{-1} \equiv A(g(z)) A(z)^{-1}, \quad \forall z \in \tilde{M} .
$$

iii) Suppose $M=\bar{M}-\left\{z_{1}, \cdots, z_{m}\right\}$ for $\bar{M}$ another Riemann surface and suppose that the singularity of $\Omega$ in $z_{j}$ is at most a simple pole. Then for $g_{j} \in \pi_{1}(M)$ corresponding to going once around $z_{j}$ in the positive direction we have that $\Lambda_{g_{j}}$ is holomorphic in $z_{j}$ and

$$
\Lambda_{g_{j}}\left(z_{j}\right)=\exp \left(2 \pi i \operatorname{Res}_{z_{j}} \Omega\right)
$$

Proof. ii) follows simply because the left hand side of (1.10) is independent of $z_{0}$.

i) follows by setting $C=I$ in (1.14) so that

$$
\Lambda(z)=A(z)^{-1} A_{\alpha} A(z)
$$

which is easily seen to satisfy (1.13).

iii) is proved in Deligne [4, théorème II, 1.17].

Remark. Let the constant matrix $T=A(g(z)) A(z)^{-1} \in N(n, \mathbb{C})$ have entries $t_{i j}$. Then $t_{i j}$ only depends on $\omega_{i+1}, \cdots, \omega_{j}$, together with the constants $c_{i^{\prime}, j^{\prime}}, i \leqq i^{\prime} \leqq j^{\prime} \leqq j$. Similar to the notation for iterated integrals we put

$$
t_{i j}=t_{g}\left(\omega_{i+1} \cdots \omega_{j}\right)
$$

(In the case $c_{i j}=0$ for $i<j$ and $g=g_{\alpha}$ for $\alpha$ a closed curve at $\pi(z)$ we clearly have $\left.t_{g}\left(\omega_{i+1} \cdots \omega_{j}\right)=\int_{\alpha} \omega_{i+1} \cdots \omega_{j}\right)$ Then (1.10) is written

$$
\int^{g z} \omega_{i+1} \cdots \omega_{j}=\sum_{i=i}^{j} t_{g}\left(\omega_{i+1} \cdots \omega_{s}\right) \int^{z} \omega_{s+1} \cdots \omega_{j}, \quad 0 \leqq i<j \leqq n
$$

similar to (1.9). 


\section{§2. Polylogarithms}

We now specialize the situation in the previous section to the following case: Let $M=\mathbb{C}-\{0,1\}$ and let $z_{0}$ lie over some point in the domain $U=\{z|| z|<1| 1-z \mid,<1\}$. Let $v_{0}$ and $v_{1}$ be the 1 -forms

$$
v_{0}=\frac{1}{2 \pi i} \frac{d z}{z}, \quad v_{1}=\frac{1}{2 \pi i} \frac{d z}{z-1} .
$$

Then as $\omega_{i}$ in the previous section we shall take either $v_{0}$ or $v_{1}$, and in order to specify a particular solution it is enough to exhibit the solution in $U$.

Case 0. Suppose $\omega_{i}=v_{0}$ for all $i=1, \cdots, n$. Then we define

$$
l_{n}^{0}(z)=\int^{z} v_{0}^{n}=\frac{1}{n !}\left(\int^{z} u_{0}\right)^{n}=\frac{1}{n !(2 \pi i)^{n}}(\log z)^{n}, \quad z \in U
$$

where $\log z$ is the usual branch of the logarithm. Notice that all integrals in the matrix $A \in N(n, \mathbb{C})$ given by $a_{i j}=\int \omega_{i+1} \cdots \omega_{j}, 0 \leqq i<j \leqq n$, are of the same form.

Case 1. Let $\omega_{1}=v_{1}$ and $\omega_{i}=v_{0}$ for $i=2, \cdots, n$. This is the case studied by Ramakrishnan [11]. We define

$$
l_{n}^{1}(z)=\int^{z} v_{1} v^{n-1}=-\frac{1}{(2 \pi i)^{n}} \operatorname{Li}_{n}(z)=-\frac{1}{(2 \pi i)^{n}} \sum_{m=1}^{\infty} \frac{z^{m}}{m^{n}}, \quad z \in U .
$$

(In particular $\mathrm{Li}_{1}(z)=-\log (1-z)$ ) Again all integrals in the matrix $A$ with $a_{i j}=\int \omega_{i+1} \cdots \omega_{j}, 0 \leqq i<j \leqq n$ are either of the form (2.2) (for $i>0$ ) or of the form (2.3).

In general for some fixed $k, 1 \leqq k \leqq n$, we shall consider

Case $k$. Let $\omega_{k}=v_{1}$ and $\omega_{i}=v_{0}$ for $i \neq k, i=1, \cdots, n$. We then define

$$
l_{n}^{k}(z)=\int^{z} v_{0}^{k-1} v_{1} v_{0}^{n-k}=\sum_{s=1}^{k}(-1)^{s+1}\left(\begin{array}{c}
n-k+s-1 \\
n-k
\end{array}\right) l_{n-k+s}^{1}(z) l_{k-s}^{0}(z) .
$$

It is straight forward to verify by induction that these functions satisfy (1.1). Again all integrals in the matrix $A$ with $a_{i j}=\int \omega_{i+1} \cdots \omega_{j}, 0 \leqq i$ $<j \leqq n$, are of the form (2.2) or (2.4) In fact 


$$
a_{i j}= \begin{cases}l_{j-i}^{0}, & \text { for } j<k \text { or } i \geqq k, \\ l_{j-i}^{k-i}, & \text { for } i<k \leqq j\end{cases}
$$

For later use let us notice the asymptotic behaviour of $l_{n}^{k}$ for $z \rightarrow 0$ and $z \rightarrow 1$ :

$$
\begin{aligned}
& \left.l_{n}^{k}(z)=O\left(|z|(-\log |z|)^{k-1}\right) \quad \text { as } z \rightarrow 0 \quad \text { (if } 1 \leqq k \leqq n\right)
\end{aligned}
$$

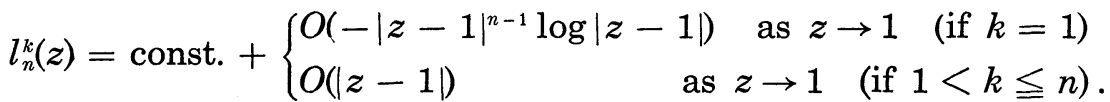

In particular

$$
\begin{array}{lll}
l_{n}^{k}(z) \rightarrow 0 & \text { for } z \rightarrow 0 \quad(\text { if } 1 \leqq k \leqq n) \\
l_{n}^{k}(z) \rightarrow \text { const. } & \text { for } z \rightarrow 1 \quad(\text { if } 1<k \text { or } 1<n) .
\end{array}
$$

We will now determine the monodromy in the above cases: Thus let $g_{0}$ and $g_{1}$ be the covering transformations of the universal covering of $\mathbb{C}-\{0,1\}$ corresponding to going once around 0 respectively 1 in the positive direction. The following generalizes Ramakrishnan [11, Theorem]:

Theorem 2.10. Given $k=0,1, \cdots, n$ let $A \in N(n, \mathbb{C})$ be the matrix $A=\left(a_{i j}\right)$ given by (2.5). Then the matrices $T_{g_{0}}, T_{g_{1}} \in N(n, \mathbb{C})$

$$
T_{g_{0}}=A\left(g_{0}(z)\right) A(z)^{-1} \quad \text { and } \quad T_{g_{1}}=A\left(g_{1}(z)\right) A(z)^{-1}
$$

are given by $T_{g_{0}}=\left(t_{i j}^{0}\right), T_{g_{1}}=\left(t_{i j}^{1}\right)$, where

$$
\begin{aligned}
t_{i j}^{0} & = \begin{cases}1 /(j-i) ! & \text { if } i \leqq j<k \text { or } k \leqq i \leqq j \\
0 & \text { otherwise }\end{cases} \\
t_{i j}^{1} & = \begin{cases}1 & \text { if } i=j \text { or } i=k-1, j=k \\
0 & \text { otherwise. }\end{cases}
\end{aligned}
$$

In particular $A$ defines a well-defined holomorphic map $A: \mathbb{C}-\{0,1\} \rightarrow$ $\Gamma \backslash N(n, \mathbb{C})$ where $\Gamma$ is a discrete subgroup of matrices with rational entries.

Proof. In the notation of Section 1 consider

$$
\Lambda_{g_{0}}=\left(\lambda_{i j}^{0}\right), \quad \Lambda_{g_{1}}=\left(\lambda_{i j}^{1}\right) .
$$

Then $\Lambda_{g_{0}}$ and $\Lambda_{g_{1}}$ are determined by Proposition 1.12. That is, they satisfy (1.13) and the initial conditions

$$
\Lambda_{g_{0}}(0)=\exp \left(2 \pi i \operatorname{Res}_{0} \Omega\right), \quad \Lambda_{g_{1}}(1)=\exp \left(2 \pi i \operatorname{Res}_{1} \Omega\right) .
$$


Then

$$
T_{g_{0}}=A \Lambda_{g_{0}} A^{-1}, \quad T_{g_{1}}=A \Lambda_{g_{1}} A^{-1} .
$$

Case $k=0$. In this case $A$ is nonsingular at 1 so that

$$
T_{g_{1}}=\Lambda_{g_{1}}=I
$$

Also $\Lambda_{g_{0}}$ is constant and

$$
\lambda_{i j}^{0}= \begin{cases}1 /(j-i) ! & \text { if } i \leqq j \\ 0 & \text { otherwise. }\end{cases}
$$

Letting $z \rightarrow 1, A(z) \rightarrow I$ so also

$$
t_{i j}^{0}= \begin{cases}1 /(j-i) ! & \text { if } i \leqq j \\ 0 & \text { otherwise. }\end{cases}
$$

Case $k>0$. The statements in (2.11) for $i \leqq j<k$ or $k \leqq i \leqq j$ follows from the case $k=0$ by the remark following Proposition 1.12. So we only need to determine $t_{i j}^{0}$ and $t_{i j}^{1}$ for $i<k \leqq j$. By (2.14)

$$
t_{i j}^{\nu}=\sum_{i \leqq r \leqq s \leqq j} a_{i r} \lambda_{r s}^{\nu} a_{s j}^{*}, \quad \nu=0,1
$$

where $A=\left(a_{i j}\right)$ is given by $(2.5)$ and $A^{-1}=\left(a_{s j}^{*}\right)$ is the inverse matrix. Notice that in (2.15) the left hand side is constant in $z$ whereas the terms on the right might vary. Also notice that the terms for $r=s$ add up to zero since $\lambda_{r r}^{\nu}=1$ and $i \neq j$. Hence

$$
t_{i j}^{\nu}=\sum_{i \leqq r<s \leqq j} a_{i r} \lambda_{r s}^{\nu} a_{s j}^{*}, \quad \nu=0,1
$$

For $\nu=1$ notice that $\lambda_{r s}^{1}$ is analytic near 1 and that by (2.13)

$$
\lambda_{r s}^{1}(1)= \begin{cases}1 & \text { if } r=s \text { or } r=k-1, j=k \\ 0 & \text { otherwise. }\end{cases}
$$

By (2.5) and (2.7) $a_{i r}$ is bounded near 1 except for $r=k, i=k-1$ where

$$
a_{k-1, k}(z)=l_{1}^{1}(z)=\frac{1}{2 \pi i} \log (1-z) .
$$

Also $a_{s j}^{*}$ grows at most as a constant times $-\log |1-z|$ as $z \rightarrow 1$, so it follows that

$$
t_{i j}^{1}=\lim _{z \rightarrow 1} a_{i k-1} a_{k j}^{*}
$$


Here if $i<k-1, a_{i k-1}=l_{k-i-1}^{0}$ has 1 as zero and similarly if $j>k, a_{k j}^{*}$ $(-1)^{j-k} l_{j-k}^{0}$ also has 1 as a zero so $t_{i j}^{1}=0$ unless $i=k-1, j=k$, in which case $t_{k-1 k}^{1}=a_{k-1 k-1} a_{k k}^{*}=1$, which proves (2.12).

For $\nu=0$ in (2.16) we have $\lambda_{r s}^{0}$ analytic near 0 and

$$
\lambda_{r s}^{0}(0)= \begin{cases}1 /(s-r) ! & \text { if } r \leqq s<k \text { or } k \leqq r \leqq s \\ 0 & \text { otherwise. }\end{cases}
$$

Also it follows from (2.5) and (2.6) that $a_{i r}$ and $a_{s j}^{*}$ grows at most like a constant times a power of $-\log |z|$ as $z \rightarrow 0$. Hence

$$
t_{i j}^{0}=\lim _{z \rightarrow 0}\left[\sum_{i \leqq r<s<k} a_{i r} a_{s j}^{*} /(s-r) !+\sum_{k \leqq r<s \leqq j} a_{i r} a_{s j}^{*} /(s-r) !\right] .
$$

Using (2.5) and (2.6) it follows that in the first term of (2.17) $a_{i r}$ grows as a constant times $(-\log |z|)^{r-i}$ whereas $a_{s j}^{*}$ grows as a constant times $|z|(-\log |z|)^{j-s-1}$, that is, the limit is zero for $z \rightarrow 0$. Similarly also the second term has limit zero for $z \rightarrow 0$ so $t_{i j}^{0}=0$. This proves the theorem.

Remark. In the notation of (1.16) we have just shown that for the iterated integrals (2.4)

$$
\begin{aligned}
& t_{g_{0}}\left(v_{0}^{n}\right)=1 / n !, \quad t_{g_{1}}\left(v_{0}^{n}\right)=0 \\
& t_{g_{0}}\left(v_{0}^{k-1} \cup_{1} v_{0}^{n-k}\right)=0 \\
& t_{g_{1}}\left(\nu^{k-1} \cup_{1} v_{0}^{n-k}\right)= \begin{cases}1 & k=n=1 \\
0 & \text { otherwise. }\end{cases}
\end{aligned}
$$

\section{§3. Shuffle relations}

Until now we have kept fixed the ordering of $\omega_{1} \cdots \omega_{n}$. We shall also need to consider simultaneously all the iterated integrals of the form $\int \omega_{i_{1}} \cdots \omega_{i_{s}}$ where $I=\left(i, \cdots, i_{s}\right)$ is a finite sequence of the numbers $1, \cdots, n$. For $I=\left(i_{1}, \cdots, i_{s}\right)$ and $J=\left(j_{1}, \cdots, j_{t}\right)$ two such sequences a shuffle of $I$ and $J$ is a sequence $K=\left(k_{1}, \cdots, k_{s+t}\right)$ such that for $s$ places $1 \leqq \nu_{1}<\nu_{2}<\ldots<\nu_{s} \leqq s+t$ we have $k_{\nu_{l}}=i_{l}, l=1, \cdots, s$, and for the remaining $t$ places $1 \leqq \mu_{1} \leqq \cdots \leqq \mu_{t} \leqq s+t, k_{\mu_{m}}=j_{m}, m=1, \cdots, t$. Following R. Ree [13] we shall say that a collection of complex numbers $\{c(I)\}, I=\left(i_{1}, \cdots, i_{s}\right)$, satisfies the shuffle relations if

$$
c\left(i_{1}, \cdots, i_{s}\right) c\left(j_{1}, \cdots, j_{t}\right)=\sum_{K} c\left(k_{1}, \cdots, k_{s+t}\right)
$$


where $K=\left(k_{1}, \cdots, k_{s+t}\right)$ runs through all shuffles of $I$ and $J$. Such a collection corresponds to a formal power series

$$
C(X)=\sum_{I} c\left(i_{1}, \cdots, i_{t}\right) X_{i_{1}} X_{i_{2}} \cdots X_{i_{s}} \quad\left(c_{\phi}=1\right)
$$

in the non-commuting variables $X_{1}, \cdots, X_{n}$, and (3.1) is satisfied if and only if $\log C(X)$ is a Lie-element (Ree [13, Theorem 2.5]). Also let recall (Ree [13, Theorem 2.4]) that the set of such power series is a group under the usual multiplication

$$
E(X)=C(X) D(X)
$$

where

$$
e\left(i_{i}, \cdots, i_{s}\right)=\sum_{\nu=0}^{s} c\left(i_{1}, \cdots, i_{\nu}\right) d\left(i_{\nu+1}, \cdots, i_{s}\right) .
$$

Finally the following observation easily follows by induction:

Proposition 3.2. Suppose the complex numbers $\{c(I)\}$ satisfy the shuffle relations (3.1). Then the iterated itegrals $\int \omega_{i_{1}} \cdots \omega_{i_{s}}, I=\left(i_{i}, \cdots, i_{s}\right)$, with initial conditions $\int^{z_{0}} \omega_{i_{1}} \cdots \omega_{i_{s}}=c\left(i_{1}, \cdots, i_{s}\right)$ also satisfy the shuffle relations, that is,

$$
\left(\int \omega_{i_{1}} \cdots \omega_{i_{s}}\right)\left(\int \omega_{j_{1}} \cdots \omega_{i_{t}}\right)=\sum_{K} \int \omega_{k_{1}} \cdots \omega_{k_{s+t}}
$$

where $K=\left(k_{1}, \cdots, k_{s+t}\right)$ runs through all shuffles of $I$ and $J$.

Remark 1. For $C(X)=\sum_{I} c(I) X_{I}$ we thus obtain a formal power series

$$
A(z)(X)=\sum_{i_{1} \cdots i_{s}}\left(\int^{z} \omega_{i} \cdots \omega_{i_{s}}\right) X_{i_{1}} \cdots X_{i_{s}}
$$

where the coefficients are holomorphic functions on $M$ with $A\left(z_{0}\right)(X)=$ $C(X)$. For $g$ a covering transformation of $\tilde{M}$ we have by (1.16):

$$
A(g z)(X)=T_{g}(X) \cdot A(z)(X)
$$

where

$$
T_{g}(X)=\sum_{i_{1} \cdots i_{s}} t_{g}\left(\omega_{i_{1}}, \cdots, \omega_{i_{s}}\right) X_{i_{1}} \cdots X_{i_{s}} .
$$

Hence we conclude that also the collection $\left\{t_{g}\left(\omega_{i_{1}} \cdots \omega_{i_{s}}\right)\right\}$ satisfies the shuffle relations, i.e. 


$$
t_{g}\left(\omega_{i_{1}} \cdots \omega_{i_{s}}\right) t_{g}\left(\omega_{j_{1}} \cdots \omega_{j_{t}}\right)=\sum_{K} t_{g}\left(\omega_{k_{1}} \cdots \omega_{k_{s+t}}\right) .
$$

Remark 2. If $\{c(I)\}$ satisfy (3.1) then they also satisfy the similar relation with more factors

$$
c\left(i_{1}, \cdots, i_{s}\right) c\left(j_{1}, \cdots, j_{t}\right) \cdots c\left(l_{1}, \cdots, l_{v}\right)=\sum_{K} c\left(k_{1}, \cdots, k_{s+t+\cdots+v}\right)
$$

with the obvious definition of shuffles of $I, J, \cdots, L$.

Remark 3. In Proposition 3.2 we considered $\int \omega_{i_{1}} \cdots \omega_{i_{s}}$ defined for all sequences $I=\left(i_{1}, \cdots, i_{s}\right)$ of numbers between 1 and $n$. In our application we shall only specify the integrals $\int \omega_{i_{1}} \cdots \omega_{i_{s}}$ for sequences $I=$ $\left(i_{1}, \cdots, i_{s}\right)$ with no repetitions. In this case the relations (3.3) and (3.4) only make sense (and are true) for $I$ and $J$ disjoint. For example in the case considered in Section 2 where $\omega_{1}=v_{1}$ and $\omega_{i}=v_{0}, i=2, \cdots, n$, we have for $I=\left(i_{1}, \cdots, i_{n}\right)$ a sequence without repetitions $\int \omega_{i_{1}} \cdots \omega_{i_{n}}=l_{n}^{k}$ for some $k$. Again (3.3) is shown by induction for disjoint sequences $I$ and $J$ using (2.6) and it follows that also (3.4) holds in this case.

We also need the following formula, which is valid just $c(I)$ is defined for sequences $I=\left(i_{1}, \cdots, i_{s}\right)$ with no repetitions.

Proposition 3.6. Suppose $\{c(I)\}$ satisfy the shuffle relations, then

$$
\begin{aligned}
\sum_{s=1}^{n}(-1)^{n-s} \sum_{1 \leqq j_{1}<j_{2}<\cdots<j_{s} \leqq n} c\left(j_{s}+1, \cdots, j_{1}\right) c\left(j_{1}+1, \cdots, j_{2}\right) & \\
\cdots c\left(j_{s-1}+1, \cdots, j_{s}\right) & =0
\end{aligned}
$$

for $n>1$. (Here $1, \cdots, n$ are taken in cyclic order).

Proof. We expand each term using (3.5) and want to determine the coefficient of $c\left(i_{1}, \cdots, i_{n}\right)$ in (3.7) for each permutation $I=\left(i_{1}, \cdots, i_{n}\right)$ of $(1, \cdots, n)$. Now a product

$$
c\left(j_{s}+1, \cdots, j_{1}\right) c\left(j_{1}+1, \cdots, j_{2}\right) \cdots c\left(j_{s-1}+1, \cdots, j_{s}\right)
$$

contributes to this coefficient if and only if $I$ is a shuffle for the $s$ sequences $\left(j_{s}+1, \cdots, j_{1}\right), \cdots,\left(j_{s-1}+1, \cdots, j_{s}\right)$. We will call the $s$-tuple $\left[j_{1}<\cdots<j_{s}\right]$ a subdivision of $(1, \cdots, n)$ and say that $I$ is $\ddot{a}$ shuffle for $\left[j_{1}<\ldots<j_{s}\right]$. The subdivisions are clearly ordered by inclusion, and it is easy to see that a given permutation $I=\left(i_{1}, \cdots, i_{n}\right)$ determines a minimal 
subdivision for which it is a shuffle. Hence if the minimal one has cardinality $k$ then $I$ is a shuffle for $\left(\begin{array}{c}n-k \\ s\end{array}\right)$ subdivisions of length $k+s$, $s=0, \cdots, n-k$. It follows that the coefficient of $c\left(i_{1}, \cdots, i_{n}\right)$ is

$$
\sum_{s=0}^{n-k}(-1)^{k+s}\left(\begin{array}{cl}
n-k \\
s
\end{array}\right)= \begin{cases}0 & \text { if } k<n \\
(-1)^{n} & \text { if } k=n .\end{cases}
$$

But if $n>1$ it is easy to see that the minimal subdivision for any permutation $I$ has length at most $n-1$ so that the coefficient of $c(I)$ is zero.

\section{§4. Tensor valued functions}

We now assume that the iterated integrals $\int \omega_{i_{1}} \cdots \omega_{i_{s}}$ satisfy Proposition 3.2 and furthermore that the monodromy constants $t_{g}\left(\omega_{i_{1}} \cdots \omega_{i_{s}}\right)$ are all rational (again it is enough to assume this for sequences $\left(i_{1}, \cdots, i_{s}\right)$ with no repetitions). In particular the expression

$$
\bar{\Phi}=\bar{\Phi}\left(\omega_{1}, \cdots, \omega_{n}\right)=\int \omega_{1} \otimes \cdots \otimes \int \omega_{n}
$$

gives a well-defined map

$$
\bar{\Phi}: M \longrightarrow \mathbb{C} / \mathbb{Q} \otimes \cdots \otimes \mathbb{C} \mathbb{Q} \quad(n \text { copies })
$$

where the tensor product is taken over $\mathbb{Q}$.

Theorem 4.2. Suppose $n>1$. Then there is a well-defined lift $\Phi$ in the diagram

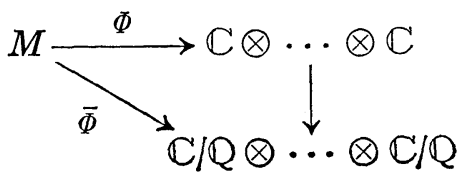

given by the expression

$$
\begin{aligned}
\Phi= & \Phi\left(\omega_{1}, \cdots, \omega_{n}\right)=\sum_{s=1}^{n}(-1)^{n-s} \sum_{1 \leqq j_{1}<\cdots<j_{s} \leqq n} \\
& \underbrace{1 \otimes \cdots \otimes 1}_{j_{1}-1} \otimes \int \omega_{j_{s+1}} \cdots \omega_{j_{1}} \otimes \underbrace{1 \otimes \cdots \otimes 1}_{j_{2-j_{1}-1}} \otimes \int \omega_{j_{1}+1} \cdots \omega_{j_{2}} \otimes \cdots \\
\cdots & \underbrace{1 \otimes \cdots \otimes}_{j_{s-j_{s-1}-1}} \otimes \int \omega_{j_{s-1+1}} \cdots \omega_{s_{s}} \otimes \underbrace{1 \otimes \cdots \otimes 1}_{n-j_{s}}
\end{aligned}
$$

(where $\omega_{1}, \cdots, \omega_{n}$ are taken in cyclic order). 
Proof. We must show that for $g$ a covering transformation of $\tilde{M}$

$$
\Phi(g z)=\Phi(z), \quad \text { for all } z \in \tilde{M} .
$$

By (1.16)

$$
\begin{aligned}
& \Phi(g z)=\sum_{s=1}^{n}(1)^{n-s} \sum_{1 \leqq j_{1}<j_{2}<\cdots<j_{s} \leqq n} \sum_{i_{1}=j_{1}}^{j_{2}} \sum_{i_{2}=j_{2}}^{j_{3}} \cdots \sum_{i_{s}=j_{s}}^{j_{1}} \\
& t_{g}\left(\omega_{j_{1}+1} \cdots \omega_{i_{1}}\right) \cdots t_{g}\left(\omega_{j_{s-1+1}} \cdots \omega_{i_{s-1}}\right) t_{g}\left(\omega_{j_{s+1}} \cdots \omega_{i_{s}}\right) \\
& \cdot \underbrace{1 \otimes \cdots \otimes 1}_{j_{1}-1} \otimes \int^{z} \omega_{i_{s+1}} \cdots \omega_{j_{1}} \otimes \underbrace{1 \otimes \cdots \otimes 1}_{j_{2}-j_{1}-1} \otimes \int^{z} \omega_{i_{1+1}} \cdots \omega_{j_{2}} \otimes \cdots \\
& \cdots \otimes \underbrace{1 \otimes \cdots \otimes 1}_{j_{s-j_{s-1}-1}} \otimes \int^{z} \omega_{i_{s-1}} \cdots \omega_{j_{s}} \otimes \underbrace{1 \otimes \cdots \otimes 1}_{n-j_{s}},
\end{aligned}
$$

where again $\omega_{1} \cdots \omega_{n}$ are taken in cyclic order. Thus each term in (4.4) corresponds to a sequence of $i$ 's and $j$ 's such that either

$$
1 \leqq j_{1} \leqq i_{1} \leqq j_{2} \leqq i_{2} \cdots \leqq j_{s} \leqq i_{s} \leqq n
$$

or

$$
1 \leqq i_{s} \leqq j_{1} \leqq i_{1} \leqq j_{2} \cdots \leqq i_{s-1} \leqq j_{s} \leqq n
$$

with strict inequality among the $j$ 's. The terms in which $j_{p}=i_{p}$ for all $p=1, \cdots, s$, are exactly the terms of $\Phi(z)$, so in the remaining terms we have $j_{p}<i_{p}$ for some $p$. In this case we have either

a) $j_{p}<i_{p}=j_{p+1}$, or

b) $j_{p}<i_{p}<j_{p+1}$.

Now any term of type b) cancels with one of type a). In fact if a sequence as in say (4.5) satisfies b) then the corresponding term cancels with the term corresponding to $j_{1}^{\prime}, i_{1}^{\prime}, \cdots, j_{s+1}^{\prime}, i_{s+1}^{\prime}$ where

$$
i_{\nu}^{\prime}=\left\{\begin{array}{ll}
i_{\nu}, & \text { for } \nu \leqq p, \\
i_{\nu-1}, & \text { for } \nu>p,
\end{array} \quad j_{\nu}^{\prime}= \begin{cases}j_{\nu}, & \text { for } \nu \leqq p \\
i_{p}, & \text { for } \nu=p, \\
j_{\nu-1}, & \text { for } \nu>p+1 .\end{cases}\right.
$$

It follows that the only non-cancelling terms of $\Phi(g z)-\Phi(z)$ are the terms in which

$$
\cdots j_{p}<i_{p}=j_{p+1}<i_{p+1}=j_{p+2} \cdots
$$

and since this is true in cyclic order we obtain $i_{p}=j_{p+1}$ for all $p=1$, $\cdots, s-1, i_{s}=j_{1}$. Hence 


$$
\begin{aligned}
\Phi(g z)-\Phi(z)= & \sum_{s=1}^{n}(-1)^{n-s} \sum_{1 \leqq j_{1}<\cdots<j_{s} \leqq n} t_{g}\left(\omega_{j_{s+1}} \cdots \omega_{j_{1}}\right) t_{g}\left(\omega_{j_{1}+1} \cdots \omega_{j_{2}}\right) \cdots \\
& \cdots t_{g}\left(\omega_{j_{s-1}+1} \cdots \omega_{j_{s}}\right) \cdot \underbrace{1 \otimes \cdots \otimes 1}_{n} \\
= & 0
\end{aligned}
$$

by Proposition 3.6. This proves the theorem.

In particular in the case of polylogarithms we use the notation $l_{n}^{k}$ of Section 2, and obtain a lift of the function $L_{n}^{*}$ defined by $(0.3)$ in the introduction:

Corollary 4.7. Suppose $n>1$. Then there is well-defined lift $L_{n}$ in the diagram

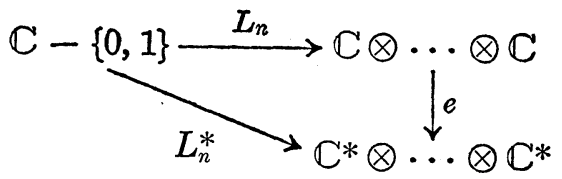

given by

$$
\begin{aligned}
& L_{n}=\sum_{s=1}^{n}(-1)^{n-s} \sum_{1 \leqq j_{1}<\cdots<j_{s} \leqq n} \underbrace{1 \otimes \cdots \otimes 1}_{j_{1}-1} \otimes l_{n-j_{s}+j_{1}}^{n-j_{s}+1} \otimes \underbrace{1 \otimes \cdots \otimes 1}_{j_{2}-j_{1}-1} \otimes \\
& \otimes l_{j_{2}-j_{1}}^{0} \otimes \cdots \otimes \underbrace{1 \otimes \cdots \otimes 1}_{j_{s-j_{s-1}-1}} \otimes l_{j_{s-j}-1}^{0} \otimes \underbrace{1 \otimes \cdots \otimes 1}_{n-j_{s}} .
\end{aligned}
$$

Remark 1. Let $\sigma$ denote the cyclic permutation of $\mathbb{C} \otimes \cdots \otimes \mathbb{C}$ given by $\sigma\left(a_{1} \otimes \cdots \otimes a_{n}\right)=a_{n} \otimes a_{1} \otimes \cdots \otimes a_{n-1}$. Then it is easy to check that

$$
\sigma \circ \Phi\left(\omega_{1}, \cdots, \omega_{n}\right)=\Phi\left(\omega_{n}, \omega_{1}, \cdots, \omega_{n-1}\right)
$$

and hence by iteration

$$
\sigma^{k} \circ \Phi\left(\omega_{1}, \cdots, \omega_{n}\right)=\Phi\left(\omega_{n-k+1}, \cdots, \omega_{n}, \omega_{1}, \cdots, \omega_{n-k}\right)
$$

for $k=0, \cdots, n-1$. In particular

$$
L_{n}^{k}=\sigma^{k-1} \circ L_{n}=\Phi\left(v_{0}, \cdots, v_{1}, \cdots, v_{0}\right), \quad k=1, \cdots, n .
$$

with $v_{1}$ on the $k$ 'th place corresponding to the " $k$ 'th case" of Section 2. Clearly $\sigma^{k} \circ L_{n}$ is a lift of

$$
L_{n}^{k *}(z)=z \otimes \cdots \otimes(1-z) \otimes \cdots \otimes z \in \mathbb{C}^{*} \otimes \cdots \otimes \mathbb{C}^{*}
$$

with $(1-z)$ on the $k$ 'th place. In the next section we shall also use the notation 


$$
\begin{aligned}
& L_{n}^{0}=\Phi\left(v_{0}, \cdots, v_{0}\right)=\sum_{s=1}^{n}(-1)^{n-s} \sum_{1 \leqq j_{1}<\cdots<j_{s} \leqq n} \\
& \underbrace{1 \otimes \cdots \otimes 1}_{j_{1}-1} \otimes l_{n-j_{s}+j_{1}}^{0} \otimes \underbrace{1 \otimes \cdots \otimes 1}_{j_{2}-j_{1}-1} \otimes l_{j_{2}-j_{1}-1}^{0} \otimes \cdots \\
& \cdots \otimes \underbrace{1 \otimes \ldots \otimes 1}_{j_{s}-j_{s-1}-1} \otimes l_{j_{s}-j_{s-1}}^{0} \otimes \underbrace{1 \otimes \cdots \otimes 1}_{n-j_{s}} .
\end{aligned}
$$

which is the lift of

$$
L_{n}^{0 *}(z)=z \otimes \cdots \otimes z \in \mathbb{C}^{*} \otimes \cdots \mathbb{C}^{*} .
$$

Remark 2. By Proposition 3.6 it clearly follows that $\Phi\left(\omega_{1}, \cdots, \omega_{n}\right)$ is in the kernel of the natural homomorphism $\mu: \mathbb{C} \otimes \cdots \otimes \mathbb{C} \rightarrow \mathbb{C}$ given by multiplication:

$$
\mu\left(a_{1} \otimes \cdots \otimes a_{n}\right)=a_{1} \cdots a_{n}
$$

\section{§. Relations}

We will finally study relations among tensor valued functions $\Phi\left(\omega_{1}, \cdots, \omega_{n}\right)$ as in (4.3) for different choices of $\omega_{i}$ 's. More precisely consider $\Psi: M \rightarrow \mathbb{C} \otimes \cdots \otimes \mathbb{C}$ of the form

$$
\Psi=\sum_{\nu=1}^{m} \Phi\left(\omega_{1}^{\nu}, \cdots, \omega_{n}^{\nu}\right)
$$

and we want to find conditions for $\Psi$ to be constant. (Relations in more than one variable can of course be reduced to this case by keeping some variables fixed). A necessary condition is of course that

$$
\bar{\Psi}=\sum_{\nu=1}^{m} \bar{\Phi}\left(\omega_{1}^{\nu}, \cdots, \omega_{n}^{\nu}\right)=\sum_{\nu=1}^{m} \int \omega_{1}^{\nu} \otimes \cdots \otimes \int \omega_{n}^{\nu}
$$

is constant in $\mathbb{C} / \mathbb{Q} \otimes \cdots \otimes \mathbb{C} / \mathbb{Q}$. As we shall see it is possible successively to work backwards from (5.2) to (5.1). First let

$$
r_{k}: \mathbb{C} \otimes \cdots \otimes \mathbb{C} \rightarrow \mathbb{C} \otimes \cdots \otimes \mathbb{C} / \mathbb{Q} \otimes \cdots \otimes \mathbb{C}
$$

be the natural reduction $\bmod \mathbb{Q}$ on the $k$ 'th factor.

Proposition 5.3. Let $\Psi: M \rightarrow \mathbb{C} \otimes \cdots \otimes \mathbb{C}$ be given by (5.1). Then $\Psi$ is constant if and only if $r_{k} \circ \Psi$ is constant for all $k=1, \cdots, n$.

Proof. Choose $z_{0} \in M$ and put $\alpha=\Psi\left(z_{0}\right)$. Then given $z \in M$,

$$
r_{k}(\Psi(z)-\alpha)=0, \quad \text { for all } k=1, \cdots, n .
$$


Hence

$$
\Psi(z)-\alpha \in \mathbb{Q} \otimes \cdots \otimes \mathbb{Q} \underset{\cong}{\stackrel{\mu}{\leftrightarrows}} \mathbb{Q}
$$

where $\mu: \mathbb{C} \otimes \cdots \otimes \mathbb{C} \rightarrow \mathbb{C}$ is given in (4.13). Now $\mu \circ(\Psi-\alpha): M \rightarrow \mathbb{C}$ is locally given by a holomorphic function and so, since it takes only rational values, must be constant. Therefore also $\Psi-\alpha$ is constant and hence 0 , so $\Psi=\alpha$.

Remark. By the last remark of Section 4 if $\Psi=\alpha$ is constant, then $\alpha$ is in the kernel of $\mu: \mathbb{C} \otimes \cdots \otimes \mathbb{C} \rightarrow \mathbb{C}$ and hence is uniquely determined by its reductions $r_{k} \alpha$.

Thus Proposition 5.3 reduces the verification of a relation among $\Phi\left(\omega_{1}^{\nu}, \cdots, \omega_{n}^{\nu}\right)$ to a relation among $r_{k} \Phi\left(\omega_{1}^{\nu}, \cdots, \omega_{n}^{\nu}\right)$ for each $k=1, \cdots, n$. Now, if we put

$$
\hat{\Phi}\left(\omega_{1}, \cdots, \omega_{n}\right)=r_{n} \Phi\left(\omega_{1}, \cdots, \omega_{n}\right) \in \mathbb{C} \otimes \cdots \otimes \mathbb{C} \otimes \mathbb{C} / \mathbb{Q}
$$

then by the Remark 1 following Corollary 4.7

$$
\sigma^{n-k} \circ r_{k} \Phi\left(\omega_{1}, \cdots, \omega_{n}\right)=\hat{\Phi}\left(\omega_{k+1}, \cdots, \omega_{n}, \omega_{1}, \cdots, \omega_{k}\right) .
$$

Hence we conclude that the conditions of Proposition 5.3 are equivalent to

$$
\sum_{\nu=1}^{m} \hat{\Phi}\left(\omega_{k+1}^{\nu}, \cdots, \omega_{n}^{\nu}, \omega_{1}^{\nu}, \cdots, \omega_{k}^{\nu}\right)=\text { const. }, \quad k=0,1, \cdots, n-1 .
$$

Here $\hat{\Phi}\left(\omega_{1}, \cdots, \omega_{n}\right)$ is given by

$$
\begin{aligned}
\hat{\Phi}\left(\omega_{1}, \cdots, \omega_{n}\right)=\sum_{s=0}^{n-1}(-1)^{n-s+1} \sum_{1 \leqq j_{1}<\cdots<j_{s} \leqq n-1} \\
\underbrace{1 \otimes \cdots \otimes 1}_{j_{1}-1} \otimes \int \omega_{1} \cdots \omega_{j_{1}} \otimes \underbrace{1 \otimes \cdots \otimes 1}_{j_{2}-j_{1}-1} \otimes \cdots \otimes \\
\underbrace{1 \otimes \cdots \otimes 1}_{n-j_{s}-1} \otimes \int \omega_{j_{s}+1} \cdots \omega_{n}
\end{aligned}
$$

where the term corresponding to $s=0$ is just

$$
1 \otimes \cdots \otimes 1 \otimes \int \omega_{1} \cdots \omega_{n}
$$

Again let

$$
r_{k}: \mathbb{C} \otimes \cdots \otimes \mathbb{C} \otimes \mathbb{C} / \mathbb{Q} \rightarrow \mathbb{C} \otimes \cdots \otimes \mathbb{C} / \mathbb{Q} \otimes \cdots \otimes \mathbb{C} \otimes \mathbb{C} / \mathbb{Q}
$$


denote the reduction $\bmod \mathbb{Q}$ on the $k$ 'th factor. Then we have (cf. Bloch [2, Corollary 6.2.3]).

TheOREm 5.9. Let $\hat{\Psi}: M \rightarrow \mathbb{C} \otimes \cdots \otimes \mathbb{C} / \mathbb{Q}$ be given by

$$
\hat{\Psi}=\sum_{\nu=1}^{m} \hat{\Phi}\left(\omega_{1}^{\nu}, \cdots, \omega_{n}^{\nu}\right)
$$

Then $\hat{\Psi}$ is constant if and only if $r_{k} \circ \hat{\Psi}$ is constant for all $k=1, \cdots, n-1$.

Proof. Choose $z_{0} \in M$ and put $\hat{\alpha}=\hat{\Psi}(z)$. We want to show that $\hat{\Psi}-\hat{\alpha}$ is constant in a neighborhood of $z_{0}$. Now by assumption for each $z \in M$

$$
\hat{\Psi}(z)-\hat{\alpha}=1 \otimes \cdots \otimes 1 \otimes a(z)
$$

and we claim that $a$ can be chosen complex analytic in a neighborhood $U$ of $z_{0}$. In fact by construction $\hat{\Psi}-\hat{\alpha}$ is represented in a suitable $U$ by such functions, i.e. we can represent

$$
\left.(\hat{\Psi}-\hat{\alpha})\right|_{U} \in A \otimes \cdots \otimes A
$$

where $A$ is the ring of analytic functions in $U$. Now if we write

$$
\left.(\hat{\Psi}-\hat{\alpha})\right|_{U}=\sum q_{i_{1} \cdots i_{n}} a_{i_{1}} \otimes \cdots \otimes a_{i_{n}}
$$

where $q_{i_{1} \cdots i_{n}} \in \mathbb{Q}$ and $a_{1}, \cdots, a_{N} \in A$ are linearly independent over $\mathbb{Q}$ then a cardinality argument shows that for some $z \in U$ also $a_{1}(z), \cdots, a_{N}(z)$ are linearly independent over $\mathbb{Q}$ from which it follows together with (5.11) that (5.12) must have the form

$$
\left.(\hat{\Psi}-\hat{\alpha})\right|_{U}=1 \otimes \cdots \otimes 1 \otimes a+\beta \otimes 1
$$

thus proving the claim. To show that $a$ is constant we shall prove that $\left.(\hat{\Psi}-\hat{\alpha})\right|_{U}$ is in the kernel of the map

$$
D: \underbrace{A \otimes \cdots \otimes A}_{n} \longrightarrow \underbrace{A \otimes \cdots \otimes A}_{n-2} \otimes \Omega_{A / \mathrm{C}}
$$

given by

$$
D\left(a_{1} \otimes \cdots \otimes a_{n}\right)=a_{1} \otimes \cdots \otimes a_{n-1} d a_{n}
$$

where $d: A \rightarrow \Omega_{A / \mathrm{C}}$ is the usual differential. This will then show that $a$ in (5.11) is in the kernel of the composite

$$
A \stackrel{1 \otimes \cdots \otimes 1 \otimes \mathrm{id}}{\longrightarrow} \underbrace{A \otimes \ldots \otimes A}_{n-1} \stackrel{\mathrm{id} \otimes d}{\longrightarrow} \underbrace{A \otimes \ldots \otimes A}_{n-2} \otimes \Omega_{A / \mathrm{C}}
$$


and since this kernel clearly consists of the constants it follows that $a$ is constant. Thus it remains to show

$$
D \hat{\Phi}\left(\omega_{1}, \cdots, \omega_{n}\right)=0
$$

where $\hat{\Phi}\left(\omega_{1}, \cdots, \omega_{n}\right)$ is given by (5.7). Now using

$$
d \int \omega_{j_{s+1}} \cdots \omega_{n}=\left(\int \omega_{j_{s+1}} \cdots \omega_{n-1}\right) \omega_{n} \quad \text { and } \quad d \int \omega_{n}=\omega_{n}
$$

we obtain

$$
\begin{aligned}
& D \hat{\Phi}=\sum_{s=0}^{n-2}(-1)^{n-s+1} \sum_{1 \leqq j_{1}<\cdots<j_{s}<n-1} \\
& \underbrace{1 \otimes \cdots \otimes 1}_{j_{1}-1} \otimes \int \omega_{1} \otimes \cdots \otimes \omega_{j_{1}} \otimes \underbrace{1 \otimes \cdots \otimes 1}_{j_{2}-j_{1}-1} \otimes \cdots \otimes \underbrace{1 \otimes \cdots \otimes 1}_{n-j_{s}-2} \\
& +\sum_{s=1}^{n-1}(-1)^{n-s+1} \sum_{1 \leqq j_{1}<\cdots<j_{s-1}<j_{s}=n-1} \\
& \underbrace{1 \otimes \cdots \otimes 1}_{j_{1}-1} \otimes \int \omega_{1} \cdots \omega_{j_{1}} \otimes \cdots \otimes \underbrace{1 \otimes \cdots \otimes 1}_{n-j_{s-1}-1} \otimes\left(\int \omega_{j_{s-1}+1} \cdots \omega_{n-1}\right) \omega_{n} \\
& =0 \\
& \otimes\left(\int \omega_{j_{s+1}} \cdots \omega_{n-1}\right) \omega_{n}
\end{aligned}
$$

which proves (5.13) and ends the proof of the theorem.

Remark. Notice that

$$
r_{k} \hat{\Phi}\left(\omega_{1}, \cdots, \omega_{n}\right)=\hat{\Phi}\left(\omega_{1}, \cdots, \omega_{k}\right) \otimes \hat{\Phi}\left(\omega_{k+1}, \cdots, \omega_{n}\right) .
$$

It follows that relations among functions of the form $\hat{\Phi}\left(\omega_{1}, \cdots, \omega_{n}\right)$ are reduced by Theorem 5.9 to relations among functions of the form $\hat{\Phi}\left(\omega, \cdots, \omega_{k}\right), k<n$.

This remark tegether with Proposition 5.3 means that any relation of the form

$$
\sum_{\nu=1}^{m} \Phi\left(\omega_{1}^{\nu}, \cdots, \omega_{n}^{\nu}\right)=\text { const. }
$$

eventually can be deduced from relations among the functions $\int \omega_{1}^{\nu}$ with values in $\mathbb{C} / \mathbb{Q}$. In particular relations among the polylogarithmic functions $L_{n}^{k}, k=0, \cdots, n$, defined in Section 4 can all be deduced from the defining relation for the ordinary logarithm

$$
\log (z+w)=\log z+\log w \quad \bmod 2 \pi i .
$$


As an illustration of this principle let us mention the following relations in one variable among the trilogarithmic functions $L^{i}=L_{3}^{i}, i=0, \cdots, 3$, defined by (4.8), (4.9), (4.11) and with values in $\mathbb{C} \otimes \mathbb{C} \otimes \mathbb{C}^{-}$:

Corollary 5.15. For $z \in \mathbb{C} \backslash\{0\}$ we have

i) $L^{1}\left(z^{-1}\right)-L^{1}(z)+L^{0}(z)=0$

ii) $L^{1}(1-z)+L^{1}\left(1-\frac{1}{z}\right)=L^{2}(z)+L^{3}(z)+L^{0}(z)$

$$
+1 \otimes 1 \otimes c-2(1 \otimes c \otimes 1)+c \otimes 1 \otimes 1
$$

where $c=-\frac{1}{(2 \pi i)^{3}} \mathrm{Li}_{3}(1)=-\frac{1}{(2 \pi i)^{3}} \sum_{k=1}^{\infty} 1 / k^{3}$.

These identities are analogous to the identities in Lewin's book [9, A.2.6, (5)-(9)]. We will not give the proof which is straight forward by successive applications of Theorem 5.9, the remark (5.14), and Proposition

5.3. The constant in ii) is determined by evaluation on $\sqrt[3]{-1}=e^{\pi i / 3}$.

From these identities one easily deduce

Corollary 5.16. For $z \in \mathbb{C}-\{0\}$ put $R(z)=L^{1}(z)-L^{2}(z)$ : Then

$$
\begin{array}{ll}
\text { i) } & R\left(z^{-1}\right)-R(z)=0 \\
\text { ii) } & R(1-z)+R\left(1-\frac{1}{z}\right)+R(z)=3(1 \otimes 1 \otimes c-1 \otimes c \otimes 1)
\end{array}
$$

It is interesting to note that relations of exactly this form naturally occur in the homology of the chain complex of configurations in the complex projective plane. We shall return to this subject and its connection with the homology of the discrete group $\operatorname{Gl}(n, \mathbb{C})$ in a paper with Sah [6] (see a]so Sah [14, Section 4]).

\section{REFERENCES}

[1] S. Bloch, Applications of the dilogarithm function in algebraic geometry, in: M. Nagata, ed., International Symposium on Algebraic Geometry, Kyoto, 1977, pp. 103114, Kinokuniya Book-Store Co., Tokyo, 1978.

[2] - Higher regulators, algebraic $K$-theory and zeta functions of elliptic curves, Lectures given at the University of California at Irvine, Preprint, 1978.

[ 3 ] K.-T. Chen, Iterated path integrals, Bull. Amer. Math. Soc., 83 (1977), 831-879.

[4] P. Deligne, Equations Différentielles à Points Singuliers Réguliers, Lecture Notes in Mathematics, 163, Springer-Verlag, Berlin-Heidelberg-New York, 1970.

[5] J. L. Dupont, The dilogarithm as a characteristic class for flat bundles, J. Pure Appl. Algebra, 44 (1987), 137-164.

[6] J. L. Dupont and C.-H. Sah, in preparation. 
[ 7 ] I. M. Gelfand and R. D. MacPherson, Geometry in Grassmannians and a generalization of the dilogarithm, Adv. in Math., 44 (1982), 279-312.

[ 8 ] R. M. Hain, The geometry of the mixed Hodge structure on the fundamental group, preprint, University of Washington, Seattle.

[9] L. Lewin, Polylogarithms and Associated Functions, North Holland, New YorkOxford, 1981.

[10] R. D. MacPherson, Grassmannian cohomology, preprint.

[11] D. Ramakrishnan, On the monodromy of higher logarithms, Proc. Amer. Math. Soc., 85 (1982), 596-599.

[12] - Analogs of the Bloch-Wigner function for higher polylogarithms, in: S. Bloch, R. K. Dennis, E. M. Friedlander, M. R. Stein, eds., Applications of Algebraic $K$-theory to Algebraic Geometry and Number Theory, Contemporary Mathematics Vol. 55, Part I, pp. 371-376, Amer. Math. Soc., Providence R. I., 1986.

[13] R. Ree, Lie elements and an algebra associated with shuffles, Ann. of Math., 68 (1958), 210-220.

[14] C.-H. Sah, Homology of classical Lie groups made discrete, III, J. Pure Appl. Algebra, 56 (1989), 269-312.

Matematisk Institut

Aarhus Universitet

Ny Munkegade

DK-8000 Aarhus $C$

Denmark 